\title{
0 baixo consumo de oxigênio tem reflexos nos escores de depressão em idosos
}

\author{
The low oxygen consumption is reflected on depression scores in elderly
}

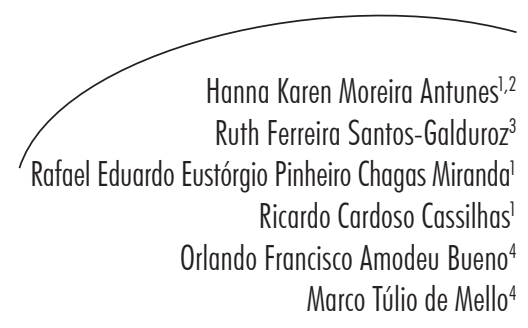

\section{Resumo}

O objetivo do estudo foi investigar os efeitos de diferentes tipos de exercício físico nos escores indicativos de depressão em idosos. Participaram 168 voluntários idosos, de ambos os sexos, com idades de 60 a 75 anos (65,66士4,28 anos), sendo 40 mulheres e 128 homens. Os voluntários foram alocados em sete grupos distintos: a) Controle sedentário ( $\mathrm{n}=40)$; b) Controle resistido ( $\mathrm{n}=23)$; c) Caminhada ( $\mathrm{n}=23)$; d) Lazer ( $\mathrm{n}=$ 20); e) Cicloergômetro ( $\mathrm{n}=23)$; f) Resistido $50 \%$ de $1 \mathrm{RM}(\mathrm{n}=19)$; e g) Resistido $80 \%$ de 1RM ( $\mathrm{n}=20)$, sendo submetidos a um protocolo de seis meses de treinamento físico, três vezes por semana. O grupo "a" permaneceu sem alterar suas atividades de rotina durante o período de estudo e não se engajou em nenhum programa de exercício físico, já o grupo "b" compareceu no laboratório, mas realizou os exercícios sem carga. Antes e imediatamente após o período de estudo, os voluntários foram submetidos a um protocolo de avaliação para determinação do consumo de oxigênio e responderam à Escala Geriátrica de Depressão. Os resultados revelaram que os grupos "c" e "e", que se exercitaram em intensidades com predomínio do metabolismo aeróbio, apresentaram redução nos escores de depressão, sendo que as demais intervenções não foram suficientes para promover redução nessa variável. Os dados sugerem que exercícios com predominância no metabolismo aeróbio são mais efetivos em promover reduções em escores de depressão de idosos.

\section{Abstract}

The study aimed to investigate the effects of different types of exercise in indicative scores of depression in the elderly. Participated 168 elderly volunteers of both sexes, aged 60-75 years $(65.66 \pm 4.28$ years), 40 women and 128 men. The volunteers were divided into seven distinct groups: a) Sedentary control $(\mathrm{n}=40)$; b) Resisted control

\footnotetext{
Centro de Estudos em Psicobiologia e Exercício. São Paulo, SP, Brasil.

Departamento de Biociências, Universidade Federal de São Paulo. Santos, SP, Brasil.

Centro de Matemática, Computação e Cognição. Universidade Federal do ABC. Santo André, SP, Brasil.

4 Departamento de Psicobiologia, Escola Paulista de Medicina. Universidade Federal de São Paulo. São Paulo, SP, Brasil.
}

Palavras-chave: Depressão. Idoso. Exercício. Consumo de Oxigênio. 
( $\mathrm{n}=23)$; c) Walking ( $\mathrm{n}=23)$; ) Outdoor ( $\mathrm{n}=20)$; e) Cycle ergometer ( $\mathrm{n}=23)$; ) Resisted $50 \%$ of 1RM ( $n=19)$; and g) Resisted $80 \%$ of 1 RM ( $n=20)$, and subjected to a protocol of six months of physical training three times a week. The group "a" remained without changing their routine activities during the study period and did not engage in any exercise program, as the group "b" appeared in the laboratory, but performed the exercises without charge. Before and immediately after the study period, the volunteers underwent an evaluation protocol for determination of oxygen consumption and responded to the Geriatric Depression Scale. The results revealed that the groups "c" and "e", who exercised at intensities with predominant aerobic metabolism, showed reduction in depression scores, and other measures were not sufficient to promote reduction in this variable. The data suggest that exercise dominance in aerobic metabolism is more effective in reducing depression scores in elderly.
Key words: Depression. Elderly. Exercise. Oxygen Consumption.

\section{INTRODUÇÃOO}

Estudos epidemiológicos têm sugerido que a depressão está associada com a inatividade física, ${ }^{1,2}$ e que essa relação é bastante evidente, particularmente em idosos. ${ }^{3}$ Além disso, observase uma diminuição desse quadro quando a atividade física é retomada. ${ }^{1}$

Embora sejam conhecidos os benefícios da prática regular do exercício físico tanto na esfera física quanto psicológica, ${ }^{4,5}$ existe ainda um número relativamente grande de indivíduos que não se engajam em nenhum tipo de atividade física regular, o que se reflete num número considerável de sedentários. Geralmente, pessoas deprimidas são fisicamente menos ativas e mais descondicionadas que indivíduos não deprimidos, ${ }^{6,7}$ no entanto, no início do quadro, questionamentos ainda permanecem se a depressão promove a inatividade física ou o contrário.

Muitos estudos têm investigado os efeitos do exercício físico no humor e encontrado resultados satisfatórios, ${ }^{8-10}$ mas devido a diferenças metodológicas entre eles, como tipo, frequência e intensidades de exercício e dúvidas em relação ao melhor tipo de exercício físico para reduzir a depressão ainda permanecem. De maneira adicional, observa-se número reduzido de trabalhos longitudinais na tentativa de elucidar o efeito do exercício físico na depressão em idosos, sendo que intervenções superiores a três meses são mais indicadas, ${ }^{11}$ para análise dos benefícios do exercício físico como ferramenta na promoção de saúde.

Neste sentido, os trabalhos envolvendo treinamento físico são, em sua maioria, direcionados para o efeito agudo do exercício físico aeróbio na redução de sintomas depressivos, tanto no âmbito profilático, ${ }^{12}$ como terapêutico, ${ }^{13}$ resultando em limitação prática no leque de opções para a população idosa.

Tendo em vista a lacuna que persiste na literatura e a importância do tema, o objetivo deste estudo foi investigar os efeitos de diferentes modelos de treinamento físico, durante seis meses, nos escores indicativos de depressão em idosos, e identificar o papel do consumo de oxigênio como índice de capacidade aeróbia interveniente nesses escores.

\section{METODOLOGIA}

\section{Procedimento ético}

Antes de participar, todos os voluntários foram informados quanto aos procedimentos, desconfortos e riscos envolvendo os processos de avaliação. Posteriormente, foi assinado um Termo de Consentimento Livre e Esclarecido para a participação voluntária no estudo. $\mathrm{O}$ protocolo experimental foi conduzido no período de março de 2002 a dezembro 2009, tendo sido 
aprovado pelo Comitê de Ética em Pesquisa da Universidade Federal de São Paulo (UNIFESP), sob o $n^{\circ}$ 207/2001, em conformidade com a Declaração de Helsinque.

\section{Voluntários}

Participaram deste estudo, 168 voluntários idosos saudáveis, de ambos os sexos, com idades entre 60 e 75 anos (65,66 44,28 anos), sendo composto por 40 mulheres e 128 homens. Os voluntários foram alocados aleatoriamente em sete grupos distintos: a) Controle sedentário $(\mathrm{n}=40) ; \mathrm{b})$ Controle resistido ( $\mathrm{n}=23)$; c) Caminhada $(\mathrm{n}=23) ; \mathrm{d})$ Lazer ( $\mathrm{n}=20)$; e) Cicloergômetro ( $\mathrm{n}=23)$; f) Resistido 50\% de 1RM ( $\mathrm{n}=19)$; e g) Resistido $80 \%$ de $1 \mathrm{RM}(\mathrm{n}=20)$.

\section{Critérios para recrutamento e seleção}

Os critérios empregados para a composição dos grupos foram: idade entre 60 e 75 anos, não ter sido submetido a intervenção cirúrgica recente e ser sedentário, isto é, não realizar nenhum tipo de exercício físico habitual. A determinação da condição de sedentarismo foi baseada em entrevista na qual foram utilizados o questionário Nivel de Atividade Física Habitual ${ }^{14}$ e uma subsequente análise do consumo de oxigênio $\left(<25 \mathrm{ml} \mathrm{kg}^{-1} \cdot \mathrm{min}^{-}\right.$ $\left.{ }^{1}\right)$. Também foram excluídos da amostra indivíduos que apresentaram alterações em exames clínicos e laboratoriais ou que fossem usuários de drogas psicoativas ou qualquer outro fármaco em que o exercício físico fosse contraindicado.

Como parte do processo seletivo e para atestar a saúde cardiovascular, os voluntários foram submetidos a um eletrocardiograma de esforço e de repouso, e aqueles considerados aptos foram inseridos no estudo. Para exclusão de quadros de demência provável, o processo de seleção também envolveu a aplicação do teste cognitivo Miniexame do Estado Mental, ${ }^{15}$ dividido em cinco subtestes: orientação, memória imediata, atenção e cálculo, recordação e linguagem, sendo adotado como nota de corte 24 pontos. $^{16}$

\section{Avaliação dos escores de depressão}

Para determinação dos escores indicativos de depressão, foi utilizada a Escala Geriátrica de Depressão (Geriatric Depression Scale, de Yesavage et $\left.a{ }^{17}{ }^{17}\right)$. Esse instrumento é utilizado para detectar depressão em pessoas idosas e oferece medidas válidas e confiáveis para a avaliação de transtornos depressivos. $O$ questionário foi aplicado individualmente em uma sala reservada, bem iluminada e ventilada. Antes de iniciar a aplicação do mesmo, os procedimentos foram explicados aos voluntários, os quais foram solicitados a respondê-los honestamente, sendo garantido total sigilo de suas respostas. Esse procedimento foi adotado em todos os voluntários antes e após o período de intervenção (seis meses), utilizando o mesmo procedimento experimental.

\section{Avaliação da capacidade aeróbia}

Os voluntários foram submetidos a uma avaliação ergoespirométrica para determinação do consumo de oxigênio pico e do limiar ventilatório 1 (LV-1). A avaliação foi conduzida em um cicloergômetro (Lifecycle 9.500HR, Schiller Park, IL, USA), utilizando o protocolo de incrementos de 25 watts a cada dois minutos, com carga inicial de três minutos a 25 watts. $\mathrm{O}$ teste foi conduzido em um analisador de gases (SensorMedics $-V_{\text {max }} 29$ series - Metabolic Measurement Cart, Yorba Linda, CA). O sistema foi précalibrado utilizando uma concentração de gases conhecidos $\left(\mathrm{O}_{2}\right.$ e $\left.\mathrm{CO}_{2}\right)$, e o fluxo foi calibrado utilizando uma seringa de três litros. Durante todo o teste, a frequência cardíaca e a pressão arterial foram monitoradas, e após atingir o consumo de oxigênio pico, o teste foi finalizado.

Com o intuito de evitar possíveis interferências circadianas, os testes foram realizados na mesma hora do dia (8-11h da manhã) em laboratório climatizado padronizado. Os critérios utilizados para determinar o LV-1 e para interromper os testes foram os descritos anteriormente na literatura. ${ }^{18-20}$ 
Intervenção

A intervenção proposta pelo estudo consistiu de seis meses de treinamento com três modalidades distintas de exercício físico (caminhada, exercício aeróbio e exercício resistido), e também seis meses com atividades de lazer. Os modelos de treinamentos utilizados seguiram as orientações da American College of Sports Medicine no que diz respeito à prescrição do treinamento físico para a população idosa. ${ }^{21,22} \mathrm{Em}$ todas as sessões de treinamento, ao longo da intervenção, os voluntários tiveram um monitoramento da pressão arterial por meio de um esfigmomanômetro e estetoscópio $\left(\right.$ Duo-sonic ${ }^{\circledR}$ ) e da frequência cardíaca por meio de um cardiofrequencímetro (Polar ${ }^{\circledR}$, modelo FS1, Kempele, Finland). A pressão arterial foi monitorada em três momentos distintos: no início do exercício, no meio da sessão de treinamento e ao término da mesma; já a frequência cardíaca foi monitorada continuamente.

\section{Descrição dos grupos}

a) Controle sedentário: o grupo controle sedentário foi composto por 40 voluntários idosos sedentários saudáveis de ambos os sexos, sendo 23 homens e 17 mulheres, com média de idade 65,42 $\pm 3,67$ anos. Esse grupo foi orientado a não alterar sua rotina de atividades diárias e a não se engajar em nenhum programa sistematizado de exercício físico durante o período de estudo (seis meses).

b) Controle resistido: o grupo controle musculação foi composto por 23 voluntários sedentários saudáveis do sexo masculino, com média de idade $66,82 \pm 2,40$ anos. Este grupo foi submetido a um período de intervenção composto por seis meses uma vez por semana, sem nenhuma sobrecarga ao longo do estudo; a metodologia para esse grupo foi similar ao descrito ao grupo " $\mathrm{f}$ ”. Durante todo o período, os voluntários desse grupo realizaram apenas o movimento de cada aparelho específico, sem carga. A inclusão desse grupo justifica- se pela tentativa de se eliminar o viés de aprendizagem neuromotora e fatores de interação social que porventura pudessem mascarar o real efeito do treinamento.

c) Caminhada: esse grupo foi composto por 23 voluntárias idosas sedentárias saudáveis do sexo feminino, com média de idade $64,04 \pm 3,19$ anos, que iniciaram um programa de condicionamento físico com predomínio no metabolismo aeróbio. O programa consistiu em sessões contínuas de 60 minutos de caminhada (duração inicial de 20 minutos com aumento gradual de volume), três vezes por semana em dias alternados durante seis meses. A intensidade do exercício físico foi prescrita mediante avaliação prévia da capacidade aeróbia. Foi utilizado como parâmetro o LV-1, utilizando a frequência cardíaca correspondente a esta intensidade. Como atividades complementares, as voluntárias realizaram exercícios de alongamento e flexibilidade articular.

d) Lazer: participaram desse grupo 20 voluntários idosos, saudáveis, de ambos os sexos, sendo dez voluntários do sexo masculino e dez do sexo feminino. A média

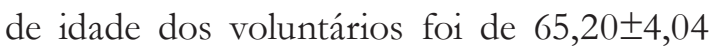
anos; eles participavam de um grupo de lazer (atividades não sistematizadas) cujas atividades eram realizadas duas vezes por semana e compostas de atividades recreativas, aulas de dança e atividades manuais.

e) Cicloergômetro: participaram desse grupo 23 voluntários idosos sedentários saudáveis do sexo masculino, com média de idade de $68,08 \pm 5,49$ anos, que iniciaram um programa de treinamento em cicloergômetro na intensidade do LV-1, três vezes por semana em dias alternados por um período de seis meses. As sessões eram contínuas, com duração inicial de 20 minutos, aumentando progressivamente para um máximo de 60 minutos. O cicloergômetro utilizado foi o Lifecycle 9.500HR, sendo determinado como parâmetro de intensidade o LV-1, utilizando 
a frequência cardíaca correspondente a esta intensidade. A carga de trabalho foi ajustada ao longo do período de estudo, de acordo com os princípios do treinamento, observando-se a relação entre volume e intensidade.

f) Resistido 50\%: participaram desse grupo 19 voluntários sedentários saudáveis do sexo masculino, com média de idade de 69,84 $\pm 4,83$ anos, que iniciaram um programa de treinamento resistido. $O$ treinamento consistiu de três sessões por semana em dias alternados com duração de 60 minutos cada sessão por um período de seis meses. A carga correspondente ao treinamento foi de $50 \%$ de uma repetição máxima (1RM) em modelo alternado por seguimento com duas séries com oito repetições para cada série. Utilizaram-se como intervalos de descansos, um minuto e 30 segundos entre as séries e três minutos entre cada aparelho. Preconizou-se nesse treinamento contemplar os principais grupos musculares utilizados pela população idosa em suas atividades de vida diária. Incluíram-se seis exercícios em aparelhos específicos do fabricante Technogym ${ }^{\circledR}$, sendo eles: chest press (adução horizontal dos ombros em rotação interna com extensão dos cotovelos, trabalhando como músculos principais o peitoral maior, tríceps e deltoides); leg press (extensão dos joelhos com extensão do quadril, trabalhando como músculos principais o quadríceps e glúteo máximo); vertical traction (adução dos ombros em rotação externa com flexão dos cotovelos [pegada pronada e pegada intermediária], trabalhando como músculos principais grande dorsal, braquiorradial e bíceps); abdominal crunch (flexão lombar, trabalhando como músculo principal o reto do abdômen); leg curl (flexão dos joelhos, trabalhando como músculo principal os Isquiotibiais) e lower back (extensão lombar, trabalhando como músculo principal os paravertebrais).

g) Resistido 80\%: participaram desse grupo 20 voluntários sedentários saudáveis do sexo

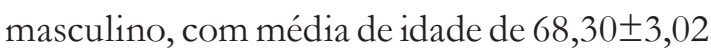

anos. O treinamento para este grupo foi similar ao grupo "f", com o diferencial de utilizar 80\% de 1RM como parâmetro de prescrição de exercício físico.

\section{Análise estatística}

Aplicou-se inicialmente o teste KolmogorovSmirnov para determinação da curva de normalidade. Para comparar as possíveis diferenças entre os grupos e entre os diferentes momentos (pré e pós-intervenção), uma análise de variância para medidas repetidas (ANOVA) com teste Duncan post-hoc foi utilizada. A correlação entre os dados de consumo de oxigênio e os escores de depressão foi feita por meio da correlação de Pearson. Os dados estão apresentados em média \pm desvio-padrão e o nível de significância adotado foi de $\mathrm{p} \leq 0,05$. A análise estatística foi conduzida com o auxílio do software Statistica Statsoft ${ }^{\circledR}$, Inc., 2004, versão 7.0.

\section{RESULTADOS}

$\mathrm{Na}$ figura 1, são apresentados os resultados da Escala Geriátrica de Depressão observada antes e após diferentes tipos de intervenção. No grupo controle sedentário, quando comparados antes e após seis meses, não foram observadas alterações significativas, mas quando os sexos foram comparados, observou-se na condição pré-período de intervenção que o sexo feminino apresentou escores maiores para depressão quando comparado ao masculino $(\mathrm{p}<0,01)$, mesmo comportamento observado na condição pós-intervenção $(\mathrm{p}<0,001)$.

No grupo submetido ao protocolo de caminhada, foi observada redução significativa dos escores de depressão, quando se compararam pré e pós-período de intervenção $(\mathrm{p}<0,005)$. Redução significativa nos escores de depressão também foi observada no grupo submetido ao protocolo na intensidade do LV1 em cicloergômetro, quando comparados pré e pósintervenção $(p<0,0001)$. No grupo submetido 
às atividades de Lazer, as comparações não revelaram diferenças significativas; esse mesmo comportamento foi observado para os grupos de Musculação 50\%, 80\% e Controle Musculação.

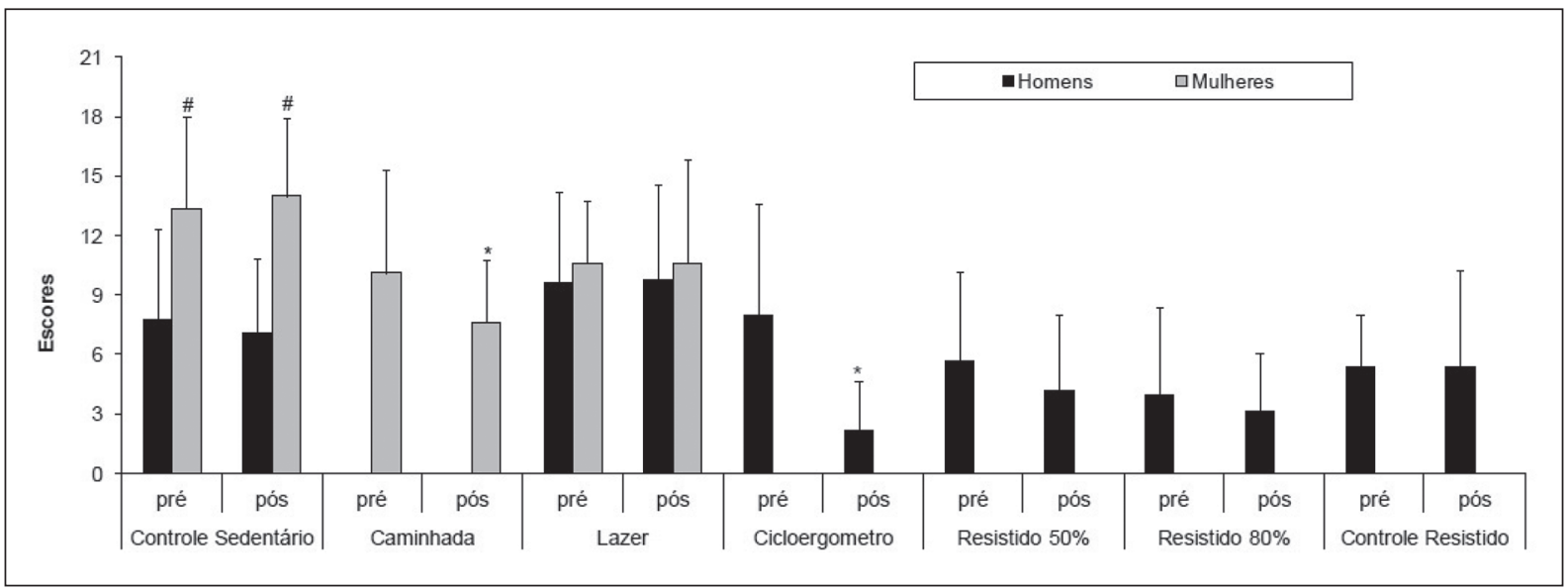

\# Diferente entre os sexos para o mesmo grupo; * diferente da condição pré-intervenção, p $\leq 0,05$.

Figura 1. Escores indicativos de depressão pré e pós-intervenção em diferentes tipos de treinamento. São Paulo-SP, 2002-2009.

$\mathrm{Na}$ figura 2, são apresentados os resultados do comportamento do consumo de oxigênio nos diferentes grupos. Na comparação pré e pósintervenção, foram observados aumentos do consumo de oxigênio nos grupos Caminhada $(\mathrm{p}<0,0001)$ e no grupo Cicloergômetro ( $\mathrm{p}<0,0001)$, e diminuição no grupo Controle Resistido ( $<<0,04)$.

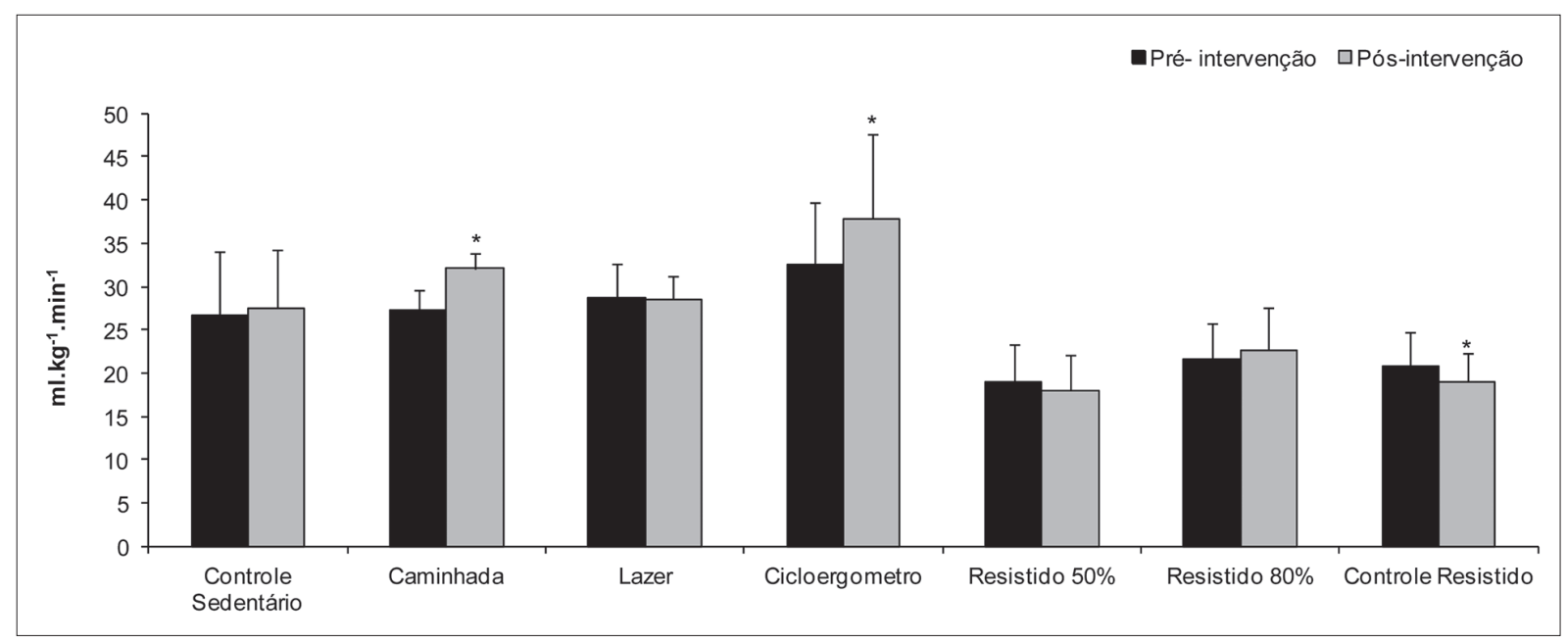

*Diferente da condição pré-intervenção, $\mathrm{p} \leq 0,05$.

Figura 2. Consumo de oxigênio pré e pós-intervenção em diferentes tipos de treinamento. São PauloSP, 2002-2009. 
$\mathrm{Na}$ figura 3, apresentam-se o resultado da correlação entre o consumo de oxigênio relativo $\left(\mathrm{ml}^{\mathrm{kg}} \mathrm{kg}^{-1} \cdot \mathrm{min}^{-1}\right)$ e os escores indicativos de depressão. Foi observada correlação média negativa entre as variáveis $(\mathrm{r}=-0,61 ; \mathrm{p}<0,05)$, mostrando que quanto maior os escores indicativos de depressão, menor o consumo de oxigênio.

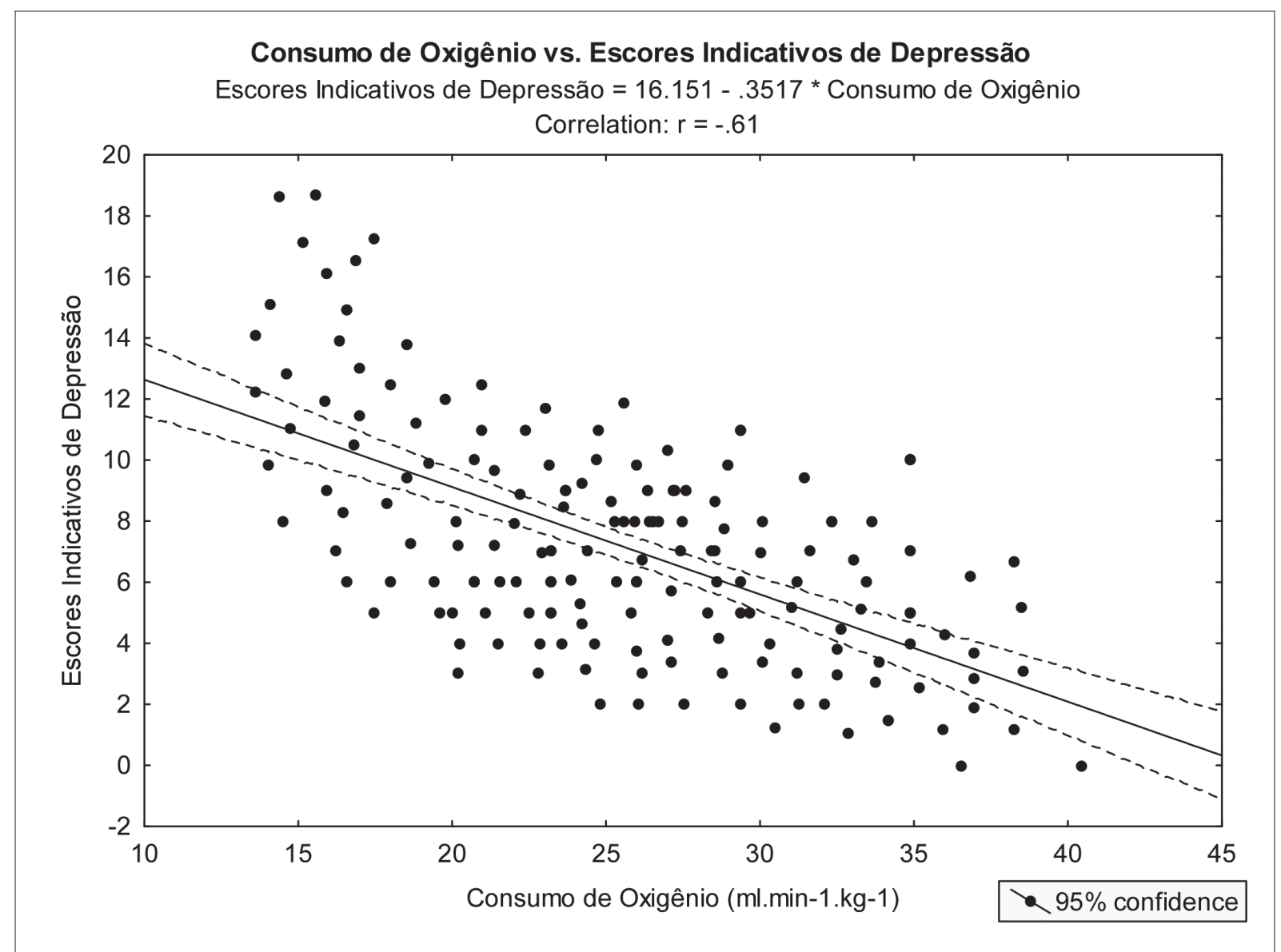

Figura 3. Correlação entre consumo de oxigênio e escores indicativos de depressão. São Paulo-SP, 2002-2009.

\section{DISCUSSÃO}

No presente estudo, foram investigados os efeitos de diferentes tipos de treinamento físico nos escores de depressão em idosos e, como resultado, encontrou-se que as intervenções executadas em intensidade moderada e com predomínio no metabolismo aeróbio, como caminhada e exercício físico realizado em cicloergômetro, ambos executados na intensidade do LV-1, ${ }^{23,24}$ tiveram os melhores resultados.
A diminuição dos escores de depressão observada nesses dois grupos que treinaram com predominância aeróbia sugere que o exercício físico aeróbio pode representar um método mais efetivo de treinamento para promover diminuição nos escores de depressão de idosos.

As hipóteses que podem auxiliar a compreender esses resultados são muitas, sendo os principais mecanismos relacionados com aspectos sociais, comportamentais, psicológico e fisiológico. ${ }^{25-28}$ Neste sentido, é possível que 
a hipótese de aumento da monoamina cerebral serotonina possa contribuir para compreender os resultados, ${ }^{29,30}$ sendo que a lipólise observada em exercícios físicos realizados na intensidade do LV-1 possa alterar a distribuição do triptofano (TRP), aminoácido precursor do neurotransmissor serotonina (relacionado com depressão). ${ }^{25,31} \mathrm{~A}$ cronicidade do treinamento também pode ter contribuir nesse sentido.

Outra possibilidade seria o exercício físico diminuir o desbalanço no eixo hipotálamopituitária-adrenal (HPA), atenuando a resposta ao estresse, ${ }^{32}$ assim como a possibilidade da participação dos opióoides endógenos. ${ }^{28,33,34}$ Além disso, recentemente tem se discutido a participação de fatores neurotróficos na redução de sintomas depressivos em processos mediados pelo exercício físico. ${ }^{35} \mathrm{O}$ BDNF (brain-derived neurotrophic factor) é forte candidato a mediar o alívio dos sintomas depressivos pelo exercício físico, devido a seu papel importante na neuroplasticidade, participação na transmissão neural, modulação, proliferação celular e neurogênese. ${ }^{36,37}$ É possível que em intensidades moderadas, como é o caso do LV-1, possa ocorrer ativação desse fator neurotrófico, ${ }^{38,39}$ que por sua vez atuaria como elemento importante e coadjuvante na terapêutica dessa condição. Há indícios também de que o BDNF é afetado pelo aumento da síntese de serotonina causada pelo exercício físico aeróbio. ${ }^{40}$

Um fator interessante no presente estudo refere-se à inclusão dos grupos-controles. Muito se discute sobre os reais efeitos do exercício físico no humor, e um dos fatores que pode ser considerado como um dos elementos importantes nessa discussão refere-se à atribuição da melhora em escores indicativos de depressão aos aspectos psicológicos e de envolvimento e interação social, uma vez que os programas de exercício permitem que o voluntário saia de casa e experimente um ambiente novo com convívio de pessoas diferentes. Esse efeito de interação pode representar um elemento de confusão na interpretação dos dados, e estudos prévios sugerem a alocação de um grupo controle que possa minimizar esse efeito. ${ }^{41,42}$
Neste estudo, trabalhou-se com gruposcontrole para os diferentes tipos de exercícios físicos com duas vertentes distintas. Um dos grupos-controle compareceu ao laboratório para realizar o gesto motor e a interação social, mas sem experimentar o efeito do treinamento físico propriamente dito, uma vez que os voluntários desse grupo não tiveram nenhum tipo de sobrecarga. Já o outro grupo-controle foi orientado a permanecer sem se engajar em nenhum tipo de atividade física, o que teoricamente privou esse grupo de comparecer em nosso laboratório e se envolver e interagir socialmente. A análise estatística não revelou diferenças quando os grupos controles foram comparados, o que sugere que os dois gruposcontrole adotados no estudo atenderam prontamente ao objetivo de ser uma referência para os grupos que se exercitaram.

O uso do exercício físico como alternativa não farmacológica é de fato importante coadjuvante na terapêutica da depressão, particularmente em idosos, por promover alívio de sintomas desagradáveis, mudanças de atitudes e modificação dos estilos de vida pouco saudáveis, o que contribui em muito com a melhoria da qualidade de vida da população. Neste sentido, as modalidades sugeridas pelo presente estudo como promotoras de redução nos escores de depressão são passíveis de realização em ambientes públicos, facilitando sua adesão e a adoção de melhores hábitos de vida.

Em conjunto, esses fatores podem representar uma economia importante para os cofres públicos, na medida em que diminuem a procura por atendimento em postos de saúde e hospitais da rede pública. Isso denota, sem dúvida, que o exercício físico regular deve ser incentivado para esta população e tal incentivo deveria ser rotina na configuração das políticas públicas de saúde no presente e no futuro.

Algumas limitações podem ser destacadas, entre elas, a não inserção do sexo feminino em todos os grupos, o que permitiria uma análise mais ampla de comparação entre os sexos. Além disso, é importante destacar a própria 
recomendação do ACSM sobre o treinamento resistido, que normatiza a prática semanal desta modalidade a apenas duas vezes por semana, limitado em duas séries, utilizada neste estudo. Certamente um maior número de estímulos, bem como maior participação semanal nesta atividade, poderiam impactar de forma positiva nos escores de depressão. Uma avaliação precisa da composição corporal, bem como a realização de análises bioquímicas séricas, contribuiriam seguramente com melhor compreensão do trabalho como um todo. Desta forma, novos estudos devem ser conduzidos com o intuito de conhecer os efeitos de diferentes tipos de exercício na depressão a partir dessas lacunas.

\section{CONCLUSÃO}

Os dados sugerem que treinamento físico com predominância no metabolismo aeróbio,

\section{REFERÊNCIAS}

1. Farmer ME, Locke BZ, Moscicki EK, Dannenberg AL, Larson DB, Radloff LS. Physical activity and depressive symptoms: the NHANES I epidemiologic follow-up study. Am J Epidemiol 1988;128(6):1340-51.

2. Camacho TC, Roberts RE, Lazarus NB, Kaplan GA, Cohen RD. Physical activity and depression: evidence from the Alameda County study. Am J Epidemiol 1991;134(2):220-31.

3. Lampinen P, Heikkinen R, Ruoppila I. Changes in intensity of physical exercise as predictors of depressive symptoms among older adults: an eightyear follow-up. Prev Med 2000;30(5):371-80.

4. Warburton DER, Nicol CW, Bredin SSD. Health benefits of physical activity: the evidence. CMAJ 2006;174(6):801-9.

5. Antunes HKM, Santos RF, Cassilhas R, Santos RVT, Bueno OFA, De Mello MT. Exercício e função cognitiva: uma revisão. Rev Bras Med Esporte 2006;12(2):108-14.

6. Martinsen EW. Benefits of exercise for the treatment of depression. Sports Med 1990;9(6):380-9.

7. Paluska SA, Schwenk TL. Physical activity and mental health: current concepts. Sports Med 2000;29(3):167-80. particularmente na intensidade referente ao limiar ventilatório 1, independentemente da modalidade cíclica, é mais efetivo em promover reduções em escores de depressão em idosos.

É importante considerar que as atividades aeróbias prescritas, realizadas três vezes por semana, e o modelo de treinamento adotado com a execução de duas séries, duas vezes por semana, podem ter contribuído com os resultados encontrados, caracterizando um modelo insuficiente para as adaptações desejadas neste estudo.

\section{AGRADECIMENTOS}

Os autores são gratos a Everald Van Cooler, por toda assistência técnica e científica, e ao suporte financeiro de AFIP, CEPE, CEMSA, CNPq, CAPES, CEPID/SONO-FAPESP (98/14303-3) e UNIFESP.

8. De Moor MHM, Beem AL, Stubbe JH, Boomsma DI, De Geus EJC. Regular exercise, anxiety, depression and personality: a population-based study. Prev Med 2006;42(4):273-9.

9. Dunn AL, Trivedi MH, Kampert JB, Clark CG, Chambliss HO. Exercise treatment for depression: efficacy and dose response. Am J Prev Med 2005;28(1):1-8.

10. Singh NA, Clements KM, Singh MAF. The efficacy of exercise as a long-term antidepressant in elderly subjects: a randomized, controlled trial. J Gerontol Ser A Biol Sci Med Sci 2001;56(8):M497-M504.

11. Forsman AK, Nordmyr J, Wahlbeck K. Psychosocial interventions for the promotion of mental health and the prevention of depression among older adults. Health Promot Int 2011;26(Supl 1);85-107.

12. Ernst C, Olson AK, Pinel JPJ, Lam RW, Christie BR. Antidepressant effects of exercise: Evidence for an adult-neurogenesis hypothesis? Rev Psychiatr 2006;31(2);84-92.

13. Vasques PE, Silveira H, Deslandes AC, Laks J. Acute exercise improves cognition in the depressed elderly: the effect of dual-tasks. Clin Sci 2011;66(9);1553-57. 
14. Baecke JA, Burema J, Frijters JE. A short questionnaire for the measurement of habitual physical activity in epidemiological studies. Am J Clin Nutr 1982;36(5):936-42.

15. Folstein MF, Folstein SE, McHugh PR. "Mini-mental state:" A practical method for grading the cognitive state of patients for the clinician. J Psychiatr Res 1975;12(3):189-98.

16. Tombaugh TN, McIntyre NJ. The mini-mental state examination: a comprehensive review. J Am Geriatr Soc 1992;40(9):922-35.

17. Yesavage JA, Brink TL, Rose TL, Lum O, Huang $\mathrm{V}$, Adey M, et al. Development and validation of a geriatric depression screening scale: a preliminary report. J Psychiatr Res 1982-1983;17(1):37-49.

18. Wasserman K, Koike A. Is the anaerobic threshold truly anaerobic? Chest. 1992;101(5 Supl):211S-18.

19. Wasserman K, Whipp BJ, Koyl SN, Beaver WL. Anaerobic threshold and respiratory gas exchange during exercise. J App Physiol 1973;35(2):236-45.

20. Wasserman K. Determinants and detection of anaerobic threshold and consequences of exercise above it. Circulation 1987;76(6 Pt 2):29-39.

21. American College of Sports Medicine position stand. The recommended quantity and quality of exercise for developing and maintaining cardiorespiratory and muscular fitness in healthy adults. Med Sci Sports Exerc 1990;22:265-74.

22. American College of Sports Medicine. Guidelines for exercise testing and prescription. Whaley $\mathrm{MH}$, Brubaker PH, Otto RM. Philadelphia, PA: Lea \& Febiger; 2006.

23. Whipp B, Ozyener F. Oxygen uptake kinetics and domains of exercise intensity. Sci Motric 1998;35$6,73-4$.

24. Whipp BJ, Özyener F. The kinetics of exertion O2 uptake: assumptions and inferences. Med Sport. 1998;51:39-49.

25. Barbour KA, Blumenthal JA. Exercise training and depression in older adults. Neurobiol Aging 2005;26(1 Supl):119-23.

26. Antunes HKM, Stella SG, Sants RF, Bueno OFA, De Mello MT. Depression, anxiety and quality of life scores in senior after an endurance exercise program. Rev Bras Psiquiatr 2005;27(4):266-71.
27. Craft LL, Perna FM. The benefits of exercise for the clinically depressed. Prim Care Companion J Clin Psychiatry 2004;6(3):104-11.

28. Brosse AL, Sheets ES, Lett HS, Blumenthal JA. Exercise and the treatment of clinical depression in adults: recent findings and future directions. Sports Med 2002;32(12):741-60.

29. Strüder HK, Weicker H. Physiology and pathophysiology of the serotonergic system and its implications on mental and physical performance. Int J Sports Med 2001;22(7 Pt 1):467-81.

30. Strüder HK, Weicker H. Physiology and pathophysiology of the serotonergic system and its implications on mental and physical performance. Int J Sports Med 2001;22(7 Pt 2):482-97.

31. Costill DL, Bowers R, Braunam G, Sparks K. Muscle Glicognen utilization during prolonged exercise on successive days. J Appl Physiol 1971;31(6):834-8.

32. Buckworth J, Dishman RK. Exercise psychology. New York: Human Kinetics; 2002.

33. Lobstein DD, Rasmussen CL. Decreases in resting plasma betaendorphin and depression scores after endurance training. J Sports Med Phys Fit 1991;31(4):543-51.

34. Janal MN, Colt EW, Clark WC, Glusman M. Pain sensitivity, mood and plasma endocrine levels in man following long-distance running: effects of naloxone. Pain 1984;19(1):13-25.

35. Bjornebekk A, Mathé AA, Brené S. The antidepressant effect of running is associated with increased hippocampal cell proliferation. Int J Neuropsychopharmacol 2005;8(3):357-68.

36. Russo-Neustadt AA, Chen MJ. Brain-derived neurotrophic factor and antidepressant activity. Curr Pharm Des 2005;11(12):1495-510.

37. Sarbadhikari SN, Saha AK. Moderate exercise and chronic stress produce counteractive effects on different areas of the brain by acting through various neurotransmitter receptor subtypes: a hypothesis. Theor Biol Med Model. 2006;3:1-18.

38. Gold SM, Schulz KH, Hartmann S, Mladek M, Lang UE, Hekkweg R, Reer R, et al. Basal serum levels and reactivity of nerve growth factor and brain-derived neurotrophic factor to standardized acute exercise in multiple sclerosis and controls. J Neuroimmunol 2003;138(1-2):99-105. 
39. Ferris LT, Williams JS, Shen CL. The effect of acute exercise on serum brain-derived neurotrophic factor levels and cognitive function. Med Sci Sports Exerc 2007;39(4):728-34.

40. Rojas Vega S, Strüder HK, Wahrmann BV, Schmidt A, Bloch W, Hollmann W. Acute BDNF and cortisol response to low intensity exercise and following ramp incremental exercise to exhaustion in humans. Brain Res 2006;1121(1):59-65.

41. Folkins $\mathrm{CH}$, Sime WE. Physical fitness training and mental health. Am Psychol 1981;36(4):373-89.

42. Morgan WP. Affective beneficence of vigorous physical activity. Med Sci Sports Exerc 1985;17(1):94-100. 